Ptolemy's (flourished AD 127-148) Almagest is a substantial and difficult work. Because it was the culmination of centuries of Greek astronomy, with its Babylonian background, and was the principal influence on astronomical theory up to the time of Copernicus and beyond, it has received the attention of every serious historian of astronomy. But, as Professor Pedersen says in his first chapter*, "it has been talked about by many, but studied seriously only by the few." His aim is "to help students of the history of astronomy to understand and appreciate Ptolemy's great and classical work.'

Since the mathematical and astronomical ideas of the Almagest are not very abstruse, the difficulties lie in the mode of presentation. Some of these difficulties were pointed out in the twelfth century by Jäbir b. Aflah in his Correction of the Almagest. Among his many complaints, he said that the principles were obscured by numerical computations, that Ptolemy's exposition was sometimes too compressed, and that the meaning was often unclear because of the chain of translators between Ptolemy and himself. These defects Professor Pedersen has completely removed. He has elucidated the ideas behind Ptolemy's demonstrations, keeping them separate from numerical manipulations; difficult passages are examined in the light of recent scholarship; and, since he has worked from Heiberg's edition of the original Greek text, neither Professor Pedersen nor his readers have to suffer from the inaccuracies and obfuscations of translators.

Much of the clarity of this book comes from the author's ruthless use of modern notation. Not only are brackets, subscripts, square-root signs, and so on, used to describe the operations Ptolemy describes verbally, but some of his fundamental ideas are expressed in terms of functions, and some of his trigonometry is represented by the modern equivalent. Thus plane triangles are often solved in the modern way - that is, without dropping perpendiculars or circumscribing circles-and the functions tan and cot

* A Survey of the Almagest. (Acta Historica Scientiarium Naturalium et Medicinalium, Volume 30.) By Olaf Pedersen. Pp. 454. (Odense University: Odense, Denmark, 1974.) Dn. kr. 200.00

\section{Ancient astronomical masterpiece}

\author{
Sorry, for copyright \\ reasons some images \\ on this page may not \\ be available online
}

Ptolemy taking the altitude of a heavenly body with a quadrant (From G. Reisch, Margarita Philosophica, Basle, 1508)

are freely used where Ptolemy made do with chords. There is usually sufficient indication of what has been done. The result is that the reader knows that Ptolemy's exact mode of thinking has not been reported, but can see his line of reasoning without being distracted by the prolixities of archaic mathematical technique. It might be thought that modernisation is sometimes taken too far. On page 164 , for instance, the mean longitude of the Moon is found by integrating its differential coefficient. While the reader may be assumed to realise that Ptolemy did not use the calculus, the modernity of the expression is startling and not really necessary. Elsewhere the anachronism is more than justified by the gain in clarity. For example, Ptolemy's calculation of lunar longitude from his general table is not easy to understand, but is fully explained in the Survey (pages 195-9) with the help of modern symbolism, particular attention being paid to Ptolemy's interpolative method of approximation. There are one or two mistakes in interpretation-I think, for instance, that the proof of the equivalence of the simple epicyclic and excentric models (pages 137-8) is mis-stated- but such lapses are rare.

In some cases, Professor Pedersen goes beyond what is written in the Almagest, but only to make the meaning clear. Several graphs of relevant functions are included to make the astronomical model under consideration easier to understand. Further aids for the reader are to be found in Appendix B, in which all the numerical parameters calculated in the Almagest are collected, and in the lists of observations. Besides a general inventory of dated observations (Appendix A), the observational data for particular astronomical models are tabulated in the appropriate place in the text.

The purely historical content of the Survey, although not so compendious as the expository, provides a useful introduction to Alexandrian astronomy and physics. There is a short chapter on Ptolemy's other works; and his reliance, in the Almagest and elsewhere, on Babylonian and Hipparchan observations and parameters is discussed as they occur. The book is introduced by a chapter on the transmission of the Almagest up to modern times. It is completed by a large and useful bibliography.

R. P. Lorch 